\title{
Vonarburg Züllig, Maya, Melchior Russ : Cronika. Eine Luzerner Chronik aus der Zeit um 1482
}

\section{Olivier Richard}

\section{OpenEdition}

\section{Journals}

Édition électronique

URL : http://journals.openedition.org/ifha/2153

DOI : $10.4000 /$ ifha. 2153

ISSN : 2198-8943

\section{Éditeur}

IFRA - Institut franco-allemand (sciences historiques et sociales)

\section{Référence électronique}

Olivier Richard, « Vonarburg Züllig, Maya, Melchior Russ : Cronika. Eine Luzerner Chronik aus der Zeit um 1482 », Revue de l'IFHA [En ligne], Date de recension, mis en ligne le 01 janvier 2010, consulté le 22 septembre 2020. URL : http://journals.openedition.org/ifha/2153 ; DOI : https://doi.org/10.4000/ifha 2153

Ce document a été généré automatiquement le 22 septembre 2020.

(C)IFHA 


\title{
Vonarburg Züllig, Maya, Melchior Russ : Cronika. Eine Luzerner Chronik aus der Zeit um 1482
}

\author{
Olivier Richard
}

1 Ce volume d'édition-commentaire est la publication d'une thèse de l'Université de Zurich de 2008, consacrée à la chronique lucernoise de Melchior Russ. Ce texte de 1482, dont il n'existe qu'un manuscrit médiéval incomplet, reprend largement le contenu des chroniques bernoises du second XVe siècle. Il constitue une œuvre typique de la Suisse de la fin du XVe siècle, déjà marquée par l'humanisme - Russ était d'ailleurs passé par les universités de Bâle et Pavie. S'il n'existe aucune trace d'une éventuelle commande de cette chronique par les autorités de Lucerne, l'œuvre de Russ est bien à placer dans la série des chroniques faites par et pour le milieu des élites urbaines. En effet, l'auteur était fils d'un secrétaire municipal de Lucerne, et lui-même, membre du Grand Conseil, scribe au tribunal de la ville, et ayant effectué pour elle plusieurs missions diplomatiques, entendait manifestement, en écrivant cette chronique, glorifier sa cité, son statut de ville d'Empire, et en particulier son importance au sein de l'alliance des confédérés. De ce point de vue, il était bien « au service de sa ville » (p. 88).

2 Son œuvre est demeurée longtemps méconnue, les positivistes lui reprochant de n'être qu'un mauvais compilateur. Comme chroniqueur de Lucerne, il avait d'ailleurs été rapidement supplanté par Diebold Schilling le jeune, qui rédigea en 1513 une chronique dont les magnifiques illustrations sont célèbres. C'est pourquoi M.V.Z. commence, dans son commentaire, par défendre l'intérêt de l'œuvre. Elle consacre peut-être un peu trop de temps à évacuer des questions désormais considérées comme secondaires, comme celle de la «valeur historique » de l'œuvre, mesurée au taux d'informations originales qu'on y trouve, mais insiste aussi sur les aspects novateurs de la chronique. Ainsi, il était visiblement prévu que les 81 pages de texte soient illustrées de 32 images, ce qui aurait fait de cette œuvre la deuxième chronique illustrée réalisée dans la Confédération. Mais finalement, les dessins ne furent jamais exécutés (« une chronique illustrée sans illustration? », p. 55). M.V.Z. entre également dans l'atelier de rédaction 
du chroniqueur, s'intéressant à ses sources et constatant qu'il était beaucoup plus à l'aise avec des textes narratifs qu'avec les chartes et autres documents d'archives.

3 L'édition du texte remplace celle de Josef Schneller, publiée en 1834 et ne correspondant plus aux exigences scientifiques actuelles. M.V.Z. a adopté le principe consistant à être le plus fidèle possible au manuscrit, y compris pour la ponctuation, qui n'est pas adaptée (aucun point !), ou pour l'emploi de majuscules/minuscules (seule exception : les noms de lieux et de personnes sont mis en majuscule. Ainsi : " So sprachen dye alten Es kement frömdt gest Also bald kam kùng Karolus... », p. T 48) ; les signes diacritiques sont tous repris - parfois transcrits sous une autre forme -, et les lignes et la pagination sont également identiques à celles du manuscrit. L'ensemble donne une édition très proche du manuscrit original, idéale pour citer le texte (objectif déclaré de M.V.Z., p. 17), mais moins pratique pour la lecture suivie. L'édition est enrichie d'une centaine de notes qui présentent des personnages mentionnés dans le texte ou donnent la date d'un événement. De belles photographies du manuscrit donnent une idée de son apparence, et un index des noms de personnes et de lieux, portant sur l'édition et le commentaire, en facilite l'accès. Nul doute que ce livre renouvellera l'intérêt pour la chronique de Melchior Russ.

Olivier Richard (Université de Haute-Alsace) 